\title{
Supermarket access, transport mode and BMI: the potential for urban design and planning policy across socio-economic areas
}

\author{
Maureen Murphy ${ }^{1, *}$, Mohammad Javad Koohsari ${ }^{2,3,4}$, Hannah Badland ${ }^{5}$ and Billie Giles-Corti ${ }^{5}$ \\ ${ }^{1}$ Centre for Health Equity, Melbourne School of Population and Global Health, The University of Melbourne, 207 \\ Bouverie Street, Melbourne, VIC 3010, Australia: ${ }^{2}$ Faculty of Sport Sciences, Waseda University, Tokyo, Japan: \\ ${ }^{3}$ Behavioural Epidemiology Laboratory, Baker IDI Heart and Diabetes Institute, Melbourne, Victoria, Australia: \\ ${ }^{4}$ Institute for Health and Ageing, Australian Catholic University, Melbourne, Victoria, Australia: ${ }^{5}$ Healthy Liveable \\ Cities Group, Centre for Urban Research, RMIT University, Melbourne, Victoria, Australia
}

Submitted 17 February 2017: Final revision received 12 July 2017: Accepted 24 July 2017: First published online 7 September 2017

\begin{abstract}
Objective: To investigate dietary intake, BMI and supermarket access at varying geographic scales and transport modes across areas of socio-economic disadvantage, and to evaluate the implementation of an urban planning policy that provides guidance on spatial access to supermarkets.

Design: Cross-sectional study used generalised estimating equations to investigate associations between supermarket density and proximity, vegetable and fruit intake and BMI at five geographic scales representing distances people travel to purchase food by varying transport modes. A stratified analysis by area-level disadvantage was conducted to detect optimal distances to supermarkets across socio-economic areas. Spatial distribution of supermarket and transport access was analysed using a geographic information system.

Setting: Melbourne, Australia.

Subjects: Adults ( $n$ 3128) from twelve local government areas (LGA) across Melbourne.

Results: Supermarket access was protective of BMI for participants in high disadvantaged areas within $800 \mathrm{~m}(P=0.040)$ and $1000 \mathrm{~m}(P=0.032)$ road network buffers around the household but not for participants in less disadvantaged areas. In urban growth area LGA, only $26 \%$ of dwellings were within $1 \mathrm{~km}$ of a supermarket, far less than $80-90 \%$ of dwellings suggested in the local urban planning policy. Low public transport access compounded disadvantage.

Conclusions: Rapid urbanisation is a global health challenge linked to increases in dietary risk factors and BMI. Our findings highlight the importance of identifying the most appropriate geographic scale to inform urban planning policy for optimal health outcomes across socio-economic strata. Urban planning policy implementation in disadvantaged areas within cities has potential for reducing health inequities.
\end{abstract}

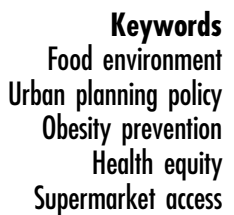

Dietary risk factors, including low vegetable and fruit intake, are among the leading risk factors for cardiovascular and circulatory disease, cancers, diabetes, and other non-communicable diseases ${ }^{(1)}$. The Global Burden of Diseases, Injuries and Risk Factors Study 2015 also singled out high BMI as presenting both a large and increasing risk for global disease burden ${ }^{(1)}$.

Increases in dietary risk factors and BMI risk are linked to economic development and processes of urbanisation, accompanied by a shift in diets to include more fats, sugars, energy-dense and processed foods, and decreasing physical $\operatorname{activity}^{(1,2)}$. However, as cities grow and become more densely populated, recent evidence suggests that urban planning can play a protective role in reducing risk exposures and noncommunicable diseases by supporting the development of health-promoting built environments ${ }^{(3,4)}$. Spatial access to food outlets, such as supermarkets and fast-food chains, is an aspect of the built environment hypothesised to influence dietary intake, BMI and non-communicable diseases ${ }^{(5,6)}$. Many studies have investigated how the location and number of food outlets in a local area influence people's health and whether spatial inequities in food outlet access contribute to dietary and health inequities ${ }^{(7)}$; however, the current evidence is limited in several ways.

First, a key challenge is the geographic scale around an individual's home at which the food environment may 
influence people's dietary and health outcomes. Few studies provide a rationale for choice of geographic scale ${ }^{(5,8)}$ and it is often acknowledged as a study limitation ${ }^{(9)}$. It has been shown that associations of food outlet access with dietary and health outcomes vary depending on how an individual's local food environment is geographically defined $^{(10,11)}$. Administrative boundaries, such as a census block or postcode, and circular or radial network buffers around a household ${ }^{(12)}$ are commonly used to model an individual's local food environment, with buffer sizes typically ranging from 400 to $4000 \mathrm{~m}^{(5)}$. Road network buffers around the household ${ }^{(12)}$ have also been applied, better representing the route an individual travels when shopping for food. A US study found that fast-food restaurants within a $1 \mathrm{~km}$ radial buffer of a household at census tract or postcode scale were detrimental to the BMI of men, however significant associations were not found at the smallest geographic scale (census block). Access to full-service restaurants, convenience stores and supermarkets was also investigated, finding significant associations with obesity risk at different levels of geographic scale for different food outlet types ${ }^{(11)}$. Another study examining the relationship between supermarket access and area-level socio-economic characteristics found that associations differed depending on the geographic scale used to define a neighbourhood ${ }^{(10)}$. Despite its importance for informing urban planning policy such as zoning regulations, geographic scale is understudied and not well understood in food environment research ${ }^{(10)}$.

Second, spatial access to food outlets differs across socio-economic areas and is impacted by an individual's transport mode ${ }^{(13)}$. Distances people travel to purchase food is influenced by their ability to access public or private transport ${ }^{(13)}$ and studies have shown that living proximally to healthy food outlets may be more important for people of low socio-economic status ${ }^{(14,15)}$. 'Food deserts', or local areas that have poor access to healthy and affordable food, may contribute to inequities in diet, BMI and non-communicable diseases ${ }^{(16)}$. In the USA, 'food deserts' in disadvantaged neighbourhoods are well documented $^{(16,17)}$ and access to a car is identified as a determinant of household access to healthy and affordable food $^{(18)}$. In other high-income countries such as Australia and the UK, the evidence for food deserts is equivo$\mathrm{cal}^{(16,19)}$. 'Deprivation amplification' describes individual or household disadvantage compounded by area-level or neighbourhood disadvantage ${ }^{(20)}$. When individuals of low socio-economic status are living in food deserts, they are exposed to a double disadvantage as their individual-level disadvantage is amplified by the area-level disadvantage of poor healthy food access ${ }^{(16)}$. If they also lack access to public transport, the area-level disadvantage is compounded further. Understanding where poor healthy food and low public transport access overlaps with area-level socio-economic disadvantage may inform efforts to reduce place-based inequities.
Several Australian studies have investigated spatial access to supermarkets across socio-economic areas ${ }^{(9,21)}$; however, these have not considered transport mode. A study conducted in forty-five suburbs in Melbourne, Australia found that participants in advantaged areas had more supermarkets within a $2 \mathrm{~km}$ road network buffer of their home and lived closer to the nearest supermarket than participants in disadvantaged areas ${ }^{(9)}$. In contrast, a Brisbane, Australia study found medium socio-economic areas had more supermarkets within a $2.5 \mathrm{~km}$ radial buffer of a census district centroid than advantaged or disadvantaged areas, and that disadvantaged areas had closest access to the nearest supermarket using a straightline distance from centroid ${ }^{(21)}$. Another study in outer Melbourne modelled travel time to supermarkets by car, bus and walking, and estimated the proportion of residents with good access using census population data. The majority of residents ( $>80 \%$ ) were found to be within an 8-10 min car journey to a supermarket, considered to be good access; and advantaged areas had closer access to supermarkets $^{(22)}$. While socio-economic areas and transport mode were considered, the study area was limited to one municipality and did not include supermarkets just outside the municipal boundary where residents are also likely to shop. Together, these studies highlight the considerable heterogeneity of food outlet access measures used to represent an individuals' food environment.

Third, there is limited evidence about the implementation of urban planning policy in relation to the food environment. Despite calls for policy evaluation to build the evidence base, few urban planning policies are evaluated for their impact on health risks and even fewer for their impact on health inequities ${ }^{(23,24)}$.

Finally, few studies have assessed built environment attributes, dietary intake, physical activity and weight status simultaneously ${ }^{(25,26)}$. The current study was informed by a conceptual framework that hypothesises the pathway through which urban planning policies influence built environment attributes in local areas, which in turn impact individual and psychosocial factors that influence dietary intake through to weight status and longterm health outcomes ${ }^{(27)}$.

The context for the study was Melbourne, Australia; however, the approach and findings have potential wider application for other cities. With $75 \%$ of the world's population expected to be living in cities by $2050^{(3)}$, it is important to understand how urban planning can support healthier food environments across diverse socioeconomic areas within cities. Melbourne, the capital of Victoria, is experiencing rapid growth and urbanisation on its perimeter, with the population expected to increase from 4.5 million in 2015 to 8.0 million in $2051^{(28)}$. Victorian planning guidelines for urban growth areas specify that $80-90 \%$ of households should be located within $1 \mathrm{~km}$ of a town centre large enough to allow for provision of a supermarket ${ }^{(29)}$. 
The aims of the current study were to: (i) investigate measures of supermarket access at a range of geographic scales to identify distances and food outlet densities most sensitive to optimal fruit and vegetable intake and BMI across strata of area-level socio-economic disadvantage; (ii) assess the spatial distribution of supermarkets across urban Melbourne by area-level socio-economic disadvantage and public and private transport access; and (iii) evaluate the implementation of an urban planning policy that provides guidance on spatial access to supermarkets.

\section{Methods}

The present study used cross-sectional data obtained from the Preventive Health Survey (PHS), a self-report population health data set with 9806 respondents collected in Victoria, Australia in 2012-13 by the Department of Health and Human Services. Data were cluster sampled from twenty-three local government areas (LGA) across Victoria. Primary approach letters for the PHS were mailed out on the Department of Health and Human Services' letterhead with a participant information sheet, followed up with a computer-assisted telephone interview survey. The aim of the PHS was to assess the prevalence of nutrition, physical activity and smoking behaviours among adults ( $\geq 18$ years) and the attitudes, beliefs and social norms that influence these behaviours ${ }^{(30)}$. In the present study, data from the twelve LGA in metropolitan Melbourne were selected, specifically those participants who resided in the Major Urban and Other Urban Sections of State, an Australian Bureau of Statistics' classification that represents urban centres with a population of 1000 persons to 100000 or more $^{(31)}$. Of the 9806 PHS respondents, 6707 (68.4\%) could be geocoded to self-reported residential addresses. Of these, 3141 respondents lived in urban areas of the metropolitan Melbourne region. Thirteen respondents lived in areas that did not have an Index of Relative SocioEconomic Disadvantage (IRSD) score assigned and therefore were removed from the analysis, leaving a final sample of 3128 participants. The PHS received ethics approval from Department of Health and Human Services and the relevant university ethics committee.

\section{Outcome variables}

The outcome variables were self-reported vegetable intake, fruit intake and BMI. Participants were asked how many servings of vegetables and fruit they typically eat per day. Self-reported height and weight data were collected. For the BMI analysis, 239 participants with missing height and/or weight data, sixteen pregnant participants and twelve participants with extreme BMI $\left(\geq 50 \mathrm{~kg} / \mathrm{m}^{2}\right)$ were excluded, consistent with previous studies ${ }^{(32,33)}$.

\section{Covariates}

All covariates were identified a priori from associations found in previous studies and the conceptual framework.
These were age, gender, education and income ${ }^{(34)}$, as well as the psychosocial and behavioural covariates detailed in the following sections. Participants with missing age and/ or education data were removed from the dietary intake ( $n$ 57) and BMI ( $n$ 36) analyses.

\section{Psychosocial covariates}

For the dietary intake analysis, knowledge, self-efficacy, habit and social norm covariates were included in the models. Evidence suggests that knowledge and selfefficacy are predictors of adult fruit and vegetable intake ${ }^{(35,36)}$. Participants were asked, 'To maintain good health, at least how many serves of vegetables (or fruit) do health experts recommend you should eat per day?' and 'How confident are you that you could: eat at least 5 serves of vegetables (2 serves of fruit) on most days?' The responses were collapsed into correct knowledge/not correct knowledge and confident/not confident. Habit is also a predictor of adult vegetable and fruit intake ${ }^{(36,37)}$. Participant responses to 'When you eat vegetable (or fruit), it's usually in the same place and at the same time of day' were collapsed into agree/not agree. Vegetable and fruit intake are significantly associated with injunctive and descriptive social norms for healthy eating. Injunctive norms refer to an individual's beliefs about what is acceptable in his/her community and descriptive norms refer to an individual's perception of the most usual behaviours of others ${ }^{(38)}$. Participant responses to 'The people who are important to you think that you eat enough vegetable (or fruit) each day' and 'Most people in my local area eat enough vegetable (or fruit) each day' were collapsed into agree/not agree.

\section{Behavioural covariates}

For the BMI analysis, fast-food intake, sweet drink intake, smoking status and physical activity were treated as covariates in the models. Frequency of fast-food consumption, frequency of sweet drink consumption and smoking status (current smoker/not a current smoker) were included. Participants were asked about the number of times they did walking, moderate physical activity and vigorous physical activity, and the average session length for each. All responses were truncated at $180 \mathrm{~min} / \mathrm{d}$, following data truncation protocols of previous studies ${ }^{(39)}$, and missing data were excluded ( $n$ 113). A physical activity variable for total activity per week was created from the truncated data by summing walking, moderate and vigorous physical activity (vigorous minutes were doubled prior to combining) minutes per week, then categorising into no activity, $<150 \mathrm{~min} /$ week and $\geq 150 \mathrm{~min} /$ week. Following guidelines that physical activity should be accrued on most days of the week ${ }^{(40)}$, a categorical variable was created: sedentary $(0 \mathrm{~min} /$ week $)$; insufficiently active $(<150 \mathrm{~min}$ or $\geq 150 \mathrm{~min}$ and $<5$ sessions $)$; and sufficiently active ( $\geq 150 \mathrm{~min}$ and $\geq 5$ sessions). 


\section{Local food environment exposure variables}

The primary exposure of interest was spatial access to supermarkets; however, measures for both supermarket and fast-food chain access were included in all models simultaneously to account for spatial co-occurrence of both outlet types ${ }^{(41,42)}$. Supermarkets provide a wide range of affordable and quality fruit and vegetables ${ }^{(43)}$, foods for a healthy diet ${ }^{(44)}$, and access to a supermarket is associated with healthier weight ${ }^{(6)}$. Access to unhealthy food outlets can adversely affect fruit and vegetable intake through increased opportunity to purchase unhealthy foods, increased exposure to unhealthy food promotion, shifts in social norms and food preferences, and lower cost of fast food per energy unit compared with fruit and vegetables $^{(43,45)}$.

\section{Food outlet locations}

A commercial data set of geocoded supermarket outlets in metropolitan Melbourne was purchased ${ }^{(46)}$. The branded supermarkets (Aldi, Coles, Costco, Foodworks, IGA, NQR and Woolworths) were verified by cross-checking against the supermarket company website and/or with Google Street View. Independent supermarkets were verified using Google Street View. Supermarket outlets were included in the data set if they existed in 2013, corresponding to the time when the PHS was collected, and comprised 742 outlets within $10 \mathrm{~km}$ of the metropolitan Melbourne boundary. Geocoded fast-food outlet data for McDonalds, Dominos, Subway, Hungry Jacks, Red Rooster and KFC were sourced from either www.zenbu.org or White/Yellow Pages listings and manually geocoded. All outlets were checked against company websites and/or with Google Street View. The fast-food chain data set comprised 648 outlets within $10 \mathrm{~km}$ of the metropolitan Melbourne boundary.

Previous studies have shown density of supermarkets to be significantly associated with fruit and vegetable intake at 2000, 3000 and $4000 \mathrm{~m}$ buffers ${ }^{(47,48)}$ and protective of BMI at $2000 \mathrm{~m}$ buffer ${ }^{(49)}$. Density of fast-food outlets has shown significant associations with obesity risk at $1000 \mathrm{~m}$ buffer $^{(11)}$. Travel-mode survey data ${ }^{(50)}$ showed that the median distance respondents in Melbourne walked to a supermarket was $660 \mathrm{~m}$, the median distance they drove was $2.7 \mathrm{~km}$, and the median distance they travelled by all modes including public transport was $2 \cdot 2 \mathrm{~km}$. Informed by these studies, we selected 800, 1000, 1600, 2000 and $3000 \mathrm{~m}$ as geographical scales to calculate food outlet access measures.

Food outlet density, proximity and presence measures were calculated for each participant using GIS (geographic information system) software. The OD Matrix tool in the Network Analyst extension was used to calculate the distance to the closest supermarket and fast-food outlet, and the supermarket and fast-food density around each participant's home, using road network buffers ranging from 800 to $3000 \mathrm{~m}$. These buffer sizes reflected the range of distances people may travel to purchase food by walking, public and private transport. Road network data were sourced from Vicmap Transport ${ }^{(51)}$ and a pedestrian road network was created by including walking and bike paths for the analyses at 800, 1000 and $1600 \mathrm{~m}$ buffers but removing freeways. Fast-food outlets at freeway service centres and at Melbourne Airport were excluded from these analyses because they are not accessible by pedestrians. A car road network was used at the 2000 and $3000 \mathrm{~m}$ buffers. Proximity measures were calculated along both the pedestrian and car road networks.

\section{Statistical analysis}

Population-weighted means and SE for participant's demographics, outcome variables and covariates were reported. Data were stratified using the IRSD, which provides an arealevel score for variables associated with disadvantage, including low income, unemployment, disability, singleparent households, low English-language proficiency and low levels of educational attainment. The IRSD aggregates the socio-economic census data of individuals within a geographic area ${ }^{(52)}$. When investigating area-level effects with an aggregated exposure such as IRSD, it is necessary to adjust for the corresponding individual-level socio-economic variables $^{(53,54)}$; therefore, we adjusted for educational attainment and household income. To achieve an approximately even participant sample in each stratum, 'high disadvantage' comprised IRSD deciles 1-3 ( $n$ 1079); 'mid disadvantage' comprised IRSD deciles 4-6 ( $n$ 1082); and 'low disadvantage' comprised IRSD deciles 7-10 ( $n$ 967). Tests of statistical significance between strata were calculated to compare means and proportions.

Generalised estimating equations were used to model the associations between dietary intake, BMI and the food outlet access measures. The clustered nature of the data was taken into account by adjusting for clustering at the LGA level. Separate models were fitted for each outcome (i.e. vegetable intake, fruit intake, BMI). Univariate associations between each covariate and the outcome were assessed before final inclusion in the model. Following this analysis fruit intake was removed from the BMI model because it was not a significant predictor. Multicollinearity of covariates and exposure variables was assessed by calculating the variance inflation factor. No multicollinearity was present, with all variance inflation factors $<2 \cdot 00$. Statistical significance was assessed at $\alpha<0 \cdot 05$. Analyses were conducted using the statistical software package Stata version 13.0.

\section{Spatial analysis}

Statistical areas level 1 (SA1) and mesh blocks (MB) are geographical areas defined by the Australian Bureau of Statistics. The SA1 administrative unit comprises approximately 400 persons $^{(55)}$ and a MB, the smallest geographical area, contains approximately thirty to sixty dwellings ${ }^{(56)}$. Using the population-weighted centroid for 
each MB, the point where distance to all households was smallest $^{(57)}$, the proportion of all MB within $1 \mathrm{~km}$ of a supermarket was calculated for all SA1 across the study area. This was represented visually in ArcGIS version 10.3.1 to depict quintiles of supermarket access across urban Melbourne. Public transport access was calculated as the proportion of $\mathrm{MB}$ in each SA1 within $400 \mathrm{~m}$ of a bus stop, or $600 \mathrm{~m}$ of a tram stop, or $800 \mathrm{~m}$ of a train station, distances found in Victorian urban planning policy to support compact and walkable neighbourhoods ${ }^{(58)}$. Private transport access was calculated using Australian Bureau of Statistics' vehicle ownership data at the SA1 level ${ }^{(59)}$. Both modes were collapsed into tertiles with the low access categories used in the analysis. Using GIS, supermarket access at $1 \mathrm{~km}$ was visualised with layers of area-level disadvantage ${ }^{(52)}$, low public transport access and low car ownership.

\section{Results}

The majority of participants were female (61\%), secondary educated (75\%), employed (52\%) and with an annual household income less than \$AU 50000 (46\%; Table 1). The mean age was 54.1 (se 0.31) years. Participants from high disadvantaged areas had a lower mean daily intake of vegetables $(P=0.001)$ and a higher mean BMI $(P=0.003)$ compared with those from less disadvantaged areas; however, there was no difference in fruit intake. Participants from high disadvantaged areas had a poorer knowledge of daily vegetable $(P=0.002)$ and fruit intake guidelines $(P<0.001)$; poorer self-efficacy for meeting daily vegetable intake $(P<0.001)$; less healthful habit strength for both vegetable and fruit intake $(P<0.001)$; and social norms related to poorer vegetable and fruit intake $(P<0 \cdot 001)$. Trends were also found for sweet drink consumption $(P=0 \cdot 048)$, physical activity $(P<0.001)$ and smoking status $(P<0.001)$ in the expected direction, with participants in high disadvantaged areas having less healthful behaviours.

Descriptions of geographic measures of supermarket and fast-food chain access by area-level disadvantage are presented in Table 2. Participants in high disadvantaged areas had better access $(P<0 \cdot 001)$ to both supermarkets and fastfood outlets for all density measures compared with less disadvantaged areas. Participants in high disadvantaged areas also lived closer to the nearest supermarket $(P<0 \cdot 001)$ and fast-food chain $(P<0.001)$ than those in less disadvantaged areas. Overall, $32 \%$ of study participants had access to a supermarket within $1 \mathrm{~km}$, ranging from $41 \%$ in high disadvantaged areas to $23 \%$ in low disadvantaged areas.

\section{Associations of supermarket access with dietary intake and BMI across area-level disadvantage}

Supermarket density and proximity were not associated with vegetable intake or fruit intake across most access measures and socio-economic areas. A statistically significant association was found for supermarket proximity and vegetable intake, and for supermarket density within the $800 \mathrm{~m}$ buffer and fruit intake; however, in both cases the effect size was too small (approximately 0.05 daily servings) to be of practical significance (see online supplementary material, Supplementary Table 1).

Supermarket access was protective of BMI for participants in the high disadvantaged areas within $800 \mathrm{~m}$ $(P=0.040)$ and $1000 \mathrm{~m}(P=0.032)$ pedestrian road network buffers, but not for participants in mid or low disadvantaged areas. For each additional supermarket within the $800 \mathrm{~m}$ buffer, mean BMI decreased by 0.34 (95\% CI $-0.66,-0.02) \mathrm{kg} / \mathrm{m}^{2}$ and within the $1000 \mathrm{~m}$ buffer, mean BMI decreased by 0.30 (95\% CI $-0.57,-0.03) \mathrm{kg} / \mathrm{m}^{2}$. There were no significant associations within the $1600 \mathrm{~m}$ pedestrian road network buffer (Table 3). Associations between supermarket access and BMI along car road network buffers within 2000 and $3000 \mathrm{~m}$ were not significant across any stratum of disadvantage (see online supplementary material, Supplemental Table 1).

\section{Spatial distribution of supermarkets and transport mode}

Figure 1 depicts the percentage of dwellings within each SA1 of urban Melbourne that have access to a supermarket within $1 \mathrm{~km}$. In urban Melbourne, $43 \%$ of dwellings were within $1 \mathrm{~km}$ of a supermarket. In growth area LGA, only $26 \%$ of dwellings were within $1 \mathrm{~km}$ of a supermarket. Figure 2 shows areas of high disadvantage in urban Melbourne that experience poor supermarket (beyond $1 \mathrm{~km}$ ) and low public transport or car access. Overall, 12\% of dwellings across Melbourne were in areas of high disadvantage and beyond $1 \mathrm{~km}$ to a supermarket. For growth area LGA, $19 \%$ of dwellings experienced this double disadvantage. Approximately half the dwellings in two of Melbourne's older outer-ring LGA had the double disadvantage of poor supermarket access and high area-level disadvantage (data not shown). When public transport access was considered, $2 \%$ of all dwellings across urban Melbourne were in high disadvantaged areas and experienced poor supermarket and low public transport access, reaching a high of $12 \%$ of dwellings in one outer-ring LGA. For growth areas, $4 \%$ of dwellings were in geographic areas of multiple disadvantages with high area-level disadvantage, poor supermarket access and public transport access. In urban Melbourne, $2 \%$ of all dwellings were in high disadvantaged areas and had poor supermarket and low car access.

\section{Sensitivity analysis}

Sensitivity analyses on significant results in the BMI model were conducted to explore: (i) whether significant associations within the 800 and $1000 \mathrm{~m}$ buffers persisted if BMI was modelled as a categorical variable, obese $\left(\geq 30 \cdot 00 \mathrm{~kg} / \mathrm{m}^{2}\right)$ compared with normal-weight and overweight (18.50-29.99 kg/m²); and (ii) whether significant 
Table 1 Descriptive statistics of participants by area-level disadvantage; adults $(n$ 3128) from twelve local government areas across Melbourne, Australia, Preventive Health Survey 2012-13

\begin{tabular}{|c|c|c|c|c|c|}
\hline & \multirow[b]{2}{*}{ Total } & \multicolumn{3}{|c|}{ Area-level disadvantage } & \multirow[b]{2}{*}{$P$ value* } \\
\hline & & $\begin{array}{c}\text { High } \\
(n \text { 1079) }\end{array}$ & $\begin{array}{c}\text { Mid } \\
\text { (n 1082) }\end{array}$ & $\begin{array}{c}\text { Low } \\
(n 967)\end{array}$ & \\
\hline \multicolumn{6}{|l|}{ Demographic characteristics } \\
\hline \multicolumn{6}{|l|}{ Age (years) } \\
\hline Mean & $54 \cdot 15$ & $56 \cdot 36$ & 53.98 & $51 \cdot 31$ & $<0.001$ \\
\hline SE & 0.31 & 0.53 & 0.53 & 0.56 & \\
\hline \multicolumn{6}{|l|}{$\operatorname{Sex}(\%)$} \\
\hline Male & 38.6 & 37.8 & $39 \cdot 6$ & $38 \cdot 3$ & \\
\hline Female & 61.4 & 62.2 & $60 \cdot 4$ & 61.7 & 0.679 \\
\hline \multicolumn{6}{|l|}{ Education (\%) } \\
\hline Primary & $4 \cdot 0$ & $6 \cdot 8$ & 3.0 & $1 \cdot 7$ & \\
\hline Secondary & 74.6 & 78.7 & $75 \cdot 9$ & $67 \cdot 8$ & \\
\hline Tertiary & 21.4 & 14.5 & $21 \cdot 1$ & 30.5 & $<0.001$ \\
\hline \multicolumn{6}{|l|}{ Employment status (\%) } \\
\hline Employed (including self-employed) & $52 \cdot 2$ & 41.8 & 55.7 & 61.5 & \\
\hline Unemployed & $4 \cdot 1$ & $5 \cdot 2$ & 3.5 & 3.5 & \\
\hline Not in labour force & 43.7 & 53.0 & $40 \cdot 8$ & $35 \cdot 0$ & $<0.001$ \\
\hline \multicolumn{6}{|l|}{ Annual household income (\%) } \\
\hline \$AU 0-49999 & 45.9 & $59 \cdot 6$ & 43.1 & 31.8 & \\
\hline \$AU 50000-79999 & $18 \cdot 1$ & 14.7 & 21.7 & $18 \cdot 1$ & \\
\hline \$AU $80000-124999$ & $16 \cdot 6$ & $10 \cdot 8$ & 17.5 & $22 \cdot 9$ & \\
\hline$\geq \$ A U 125000$ & 9.9 & 3.8 & $9 \cdot 4$ & $18 \cdot 2$ & \\
\hline Refused/don't know & 9.5 & $11 \cdot 1$ & 8.4 & $9 \cdot 1$ & $<0.001$ \\
\hline \multicolumn{6}{|l|}{ Outcome variables } \\
\hline \multicolumn{6}{|l|}{ Vegetable consumption (servings/d) } \\
\hline Mean & $2 \cdot 26$ & $2 \cdot 13$ & $2 \cdot 29$ & $2 \cdot 40$ & 0.001 \\
\hline SE & 0.03 & 0.05 & 0.05 & 0.05 & \\
\hline \multicolumn{6}{|l|}{ Fruit consumption (servings/d) } \\
\hline Mean & 1.74 & 1.75 & 1.73 & 1.74 & 0.911 \\
\hline SE & 0.02 & 0.04 & 0.04 & 0.04 & \\
\hline BMI $\left(\mathrm{kg} / \mathrm{m}^{2}\right)$ & & & & & \\
\hline Mean & $27 \cdot 34$ & $27 \cdot 58$ & $27 \cdot 55$ & $26 \cdot 80$ & 0.003 \\
\hline SE & 0.10 & 0.18 & 0.18 & 0.18 & \\
\hline BMI category (\%) & & & & & \\
\hline Underweight $\left(<18.50 \mathrm{~kg} / \mathrm{m}^{2}\right)$ & $1 \cdot 3$ & 1.7 & $1 \cdot 0$ & $1 \cdot 1$ & \\
\hline Normal weight $\left(18.50-24.99 \mathrm{~kg} / \mathrm{m}^{2}\right)$ & $36 \cdot 4$ & 33.1 & $36 \cdot 1$ & 40.7 & \\
\hline Overweight $\left(25.00-29.99 \mathrm{~kg} / \mathrm{m}^{2}\right)$ & $35 \cdot 3$ & $37 \cdot 1$ & $34 \cdot 4$ & $34 \cdot 6$ & \\
\hline Obese $\left(\geq 30.00 \mathrm{~kg} / \mathrm{m}^{2}\right)$ & $27 \cdot 0$ & $28 \cdot 1$ & 28.5 & 23.6 & 0.017 \\
\hline Covariates: psychosocial & & & & & \\
\hline Knowledge of RDI (\%) & & & & & \\
\hline Correct knowledge, vegetable & $27 \cdot 8$ & $24 \cdot 0$ & 28.6 & $31 \cdot 3$ & 0.002 \\
\hline Correct knowledge, fruit & $72 \cdot 5$ & $67 \cdot 1$ & 73.5 & 78.0 & $<0.001$ \\
\hline Self-efficacy (\%) & & & & & \\
\hline Eating RDI vegetable & $73 \cdot 3$ & $68 \cdot 3$ & $75 \cdot 0$ & $77 \cdot 6$ & $<0.001$ \\
\hline Eating RDI fruit & $92 \cdot 8$ & $92 \cdot 1$ & 92.5 & 93.8 & 0.336 \\
\hline Habit strength (\%) & & & & & \\
\hline Vegetable consumption & 83.0 & 78.7 & $84 \cdot 8$ & 86.4 & $<0.001$ \\
\hline Fruit consumption & $58 \cdot 2$ & 53.8 & 58.9 & $62 \cdot 8$ & $<0.001$ \\
\hline Social norms: injunctive (\%) & & & & & \\
\hline Vegetable consumption & $69 \cdot 5$ & $66 \cdot 7$ & $70 \cdot 6$ & 71.9 & 0.033 \\
\hline Fruit consumption & $65 \cdot 0$ & 62.5 & $66 \cdot 4$ & $66 \cdot 7$ & 0.082 \\
\hline Social norms: descriptive (\%) & & & & & \\
\hline Vegetable consumption & 33.9 & $32 \cdot 1$ & 31.1 & $39 \cdot 4$ & $<0.001$ \\
\hline Fruit consumption & $33 \cdot 4$ & $29 \cdot 3$ & $31 \cdot 0$ & $41 \cdot 1$ & $<0.001$ \\
\hline Covariates: behavioural & & & & & \\
\hline Fast-food consumption (frequency/fortnight) & & & & & \\
\hline Mean & $1 \cdot 12$ & $1 \cdot 15$ & 1.08 & 1.14 & 0.709 \\
\hline SE & 0.04 & 0.08 & 0.05 & 0.07 & \\
\hline Sweet drink consumption (frequency/fortnigh & & & & & \\
\hline Mean & $6 \cdot 12$ & $6 \cdot 66$ & $6 \cdot 13$ & 5.51 & 0.048 \\
\hline SE & 0.20 & 0.35 & 0.36 & 0.31 & \\
\hline Physical activity (\%) & & & & & \\
\hline Inactive & $6 \cdot 8$ & 8.4 & $6 \cdot 3$ & $5 \cdot 6$ & \\
\hline Insufficient activity (frequency \& duration) & $25 \cdot 0$ & 27.5 & $26 \cdot 3$ & $20 \cdot 0$ & \\
\hline Sufficient activity (frequency \& duration) & $68 \cdot 2$ & $64 \cdot 1$ & 67.4 & 74.4 & $<0.001$ \\
\hline Smoking status (\%) & & & & & \\
\hline Current smoker & 14.7 & $17 \cdot 4$ & $15 \cdot 1$ & 11.0 & \\
\hline Not a current smoker & $85 \cdot 3$ & 82.6 & 84.9 & $89 \cdot 0$ & $<0.001$ \\
\hline
\end{tabular}

RDI, recommended daily intake.

${ }^{*} P$ values determined by the adjusted Wald test for continuous variables and by the $X^{2}$ test for categorical variables. 
Table 2 Geographic measures of supermarket and fast-food chain access by area-level disadvantage among adults $(n$ 3128) from twelve local government areas across Melbourne, Australia, Preventive Health Survey 2012-13

\begin{tabular}{|c|c|c|c|c|c|c|c|c|c|}
\hline & & & \multicolumn{6}{|c|}{ Area-level disadvantage } & \multirow[b]{3}{*}{$P$ value* } \\
\hline & \multicolumn{2}{|c|}{ Total } & \multicolumn{2}{|c|}{ High $(n$ 1079) } & \multicolumn{2}{|c|}{ Mid $(n$ 1082) } & \multicolumn{2}{|c|}{ Low $(n 967)$} & \\
\hline & Mean & SD & Mean & SD & Mean & SD & Mean & SD & \\
\hline \multicolumn{10}{|l|}{ Supermarket density (pedestrian road network) } \\
\hline$\leq 800 \mathrm{~m}$ & 0.30 & 0.67 & 0.41 & 0.78 & 0.26 & 0.60 & 0.22 & 0.59 & $<0.001$ \\
\hline$\leq 1000 \mathrm{~m}$ & 0.49 & 0.86 & 0.68 & 1.02 & 0.43 & 0.75 & 0.36 & 0.76 & $<0.001$ \\
\hline$\leq 1600 \mathrm{~m}$ & 1.39 & 1.44 & $1 \cdot 74$ & 1.63 & $1 \cdot 32$ & 1.31 & 1.08 & $1 \cdot 27$ & $<0.001$ \\
\hline \multicolumn{10}{|l|}{ Supermarket density (car road network) } \\
\hline$\leq 2000 \mathrm{~m}$ & $2 \cdot 11$ & 1.79 & 2.55 & 1.90 & 2.02 & 1.67 & 1.71 & 1.69 & $<0.001$ \\
\hline$\leq 3000 \mathrm{~m}$ & 4.57 & 2.91 & $5 \cdot 31$ & $2 \cdot 63$ & 4.46 & 2.75 & 3.85 & $3 \cdot 19$ & $<0.001$ \\
\hline \multicolumn{10}{|c|}{ Fast-food chain density (pedestrian road network) } \\
\hline$\leq 800 \mathrm{~m}$ & 0.24 & 0.68 & 0.36 & 0.85 & 0.23 & 0.65 & 0.12 & 0.45 & $<0.001$ \\
\hline$\leq 1000 \mathrm{~m}$ & 0.43 & 0.94 & 0.62 & $1 \cdot 13$ & 0.39 & 0.87 & 0.24 & 0.71 & $<0.001$ \\
\hline$\leq 1600 \mathrm{~m}$ & 1.29 & 1.67 & $1 \cdot 74$ & 1.90 & 1.24 & 1.56 & 0.85 & 1.35 & $<0.001$ \\
\hline \multicolumn{10}{|l|}{ Fast-food chain density (car road network) } \\
\hline \multirow{3}{*}{$\leq 3000 \mathrm{~m}$} & $2 \cdot 00$ & 2.03 & 2.57 & $2 \cdot 15$ & 1.93 & 1.98 & 1.43 & 1.76 & $<0.001$ \\
\hline & 4.49 & $2 \cdot 82$ & $5 \cdot 31$ & $2 \cdot 70$ & 4.41 & $2 \cdot 80$ & 3.66 & $2 \cdot 73$ & $<0.001$ \\
\hline & Median & IQR & Median & IQR & Median & IQR & Median & IQR & \\
\hline $\begin{array}{l}\text { Distance to closest supermarket (pedestrian } \\
\text { road network) }(\mathrm{km})\end{array}$ & $1 \cdot 30$ & $0.88-1.80$ & $1 \cdot 12$ & $0.78-1.61$ & 1.33 & $0.91-1.83$ & 1.46 & $1.04-1.99$ & $<0.001$ \\
\hline $\begin{array}{l}\text { Distance to closest fast-food chain (pedestrian } \\
\text { road network) }(\mathrm{km})\end{array}$ & 1.55 & $1.03-2 \cdot 19$ & 1.29 & $0.89-1.87$ & 1.58 & $1 \cdot 06-2 \cdot 22$ & 1.74 & $1 \cdot 26-2.47$ & $<0.001$ \\
\hline
\end{tabular}

IQR, interquartile range.

${ }^{*} P$ values determined by ANOVA for food outlet density and Kruskal-Wallis test for food outlet proximity.

results within the $1000 \mathrm{~m}$ buffer were due to the influence of supermarkets within the $800 \mathrm{~m}$ buffer. First, when BMI was modelled as a categorical variable, supermarket density was associated with an approximately $15 \%$ lower odds of obesity within both $800 \mathrm{~m}(\mathrm{OR}=0.86 ; 95 \% \mathrm{CI} 0.78,0.95)$ and $1000 \mathrm{~m}$ $(\mathrm{OR}=0.85 ; 95 \% \mathrm{CI} 0.76,0.96)$ pedestrian road network buffers for participants living in high disadvantaged areas. Second, a 'donut-buffer' exposure measure was created that counted the supermarkets within 801-1000 m of participants' address, following the method described in a previous study ${ }^{(60)}$. The protective effect of supermarkets for people in disadvantaged areas persisted for participants within the $800 \mathrm{~m}$ pedestrian road network buffer of a supermarket but was not found for the 801-1000 $\mathrm{m}$ donut-buffer (see online supplementary material, Supplemental Table 2).

\section{Discussion}

The current study examined dietary intake, BMI and supermarket access at a range of geographic scales across areas of socio-economic disadvantage in urban Melbourne, Australia. Participants in high disadvantaged areas had lower vegetable intake and higher BMI compared with those in less disadvantaged areas, consistent with previous studies that find a social gradient in health risks and outcomes ${ }^{(61)}$. Participants in high disadvantaged areas had better access to supermarkets and fast-food outlets than those in less disadvantaged areas, a result that contrasts with the two earlier Melbourne studies where participants in disadvantaged areas had poorer supermarket access ${ }^{(9,22)}$. However, given the present study was conducted across a larger study area and comprised 742 supermarkets, compared with fifteen and seventy-four supermarkets in the previous studies ${ }^{(9,22)}$, our findings may more comprehensively and accurately represent supermarket access in urban Melbourne.

Supermarket density at 800 and $1000 \mathrm{~m}$ was protective of BMI for participants in high disadvantaged areas but not for those in mid or low disadvantaged areas. These results highlight the importance of investigating geographic scale and considering its differential impact across communities of socio-economic disadvantage. The effect size was greatest at the $800 \mathrm{~m}$ network buffer, which approximates a walkable distance of $10 \mathrm{~min}$ for adults ${ }^{(62)}$ and is close to the median distance we identified a priori that people in Melbourne walk to supermarkets ${ }^{(50)}$. Sensitivity analysis suggests that supermarkets within the $800 \mathrm{~m}$ buffer may also be responsible for the significant association between supermarkets and BMI at the larger $1000 \mathrm{~m}$ buffer. Other studies have noted that proximal access to supermarkets may be more important for people in disadvantaged areas because they are less mobile and have access to fewer resources, including income, transport ${ }^{(15)}$ and time ${ }^{(63)}$. Our findings support the hypothesis that close access to supermarkets in disadvantaged areas provides opportunities to regularly purchase affordable fresh foods while reducing reliance on transport modes other than walking. 
Counterintuitively, supermarket access was not associated with vegetable and fruit intake at a level of practical significance at any geographic scale or stratum of disadvantage. These results are consistent with a previous review that found supermarket access was consistently associated with lower BMI but not dietary intake ${ }^{(25)}$. It is possible that the null findings may be explained by the complexities in measuring dietary intake when it relies on participants' accurate recall $^{(25,43)}$. Further, a larger sample and more comprehensive measures of a healthy diet may be required to detect associations. For example, a recent study of more than 9000 adults found significant associations between supermarket access and diet quality ${ }^{(64)}$. Future research could also include additional measures of dietary intake consistent with dietary guidelines including grains, lean meat and dairy ${ }^{(65)}$. However, the BMI analysis produced an expected result, possibly because the model comprehensively captured and adjusted for multiple influences impacting weight status, including intakes of fast foods and sweet drinks, smoking status and physical activity ${ }^{(25)}$. While the present study was concerned primarily with the influence of supermarket access on dietary intake and BMI, the gradient in the knowledge and selfefficacy (vegetables) covariates across socio-economic strata is important to note (Table 1), representing an opportunity for targeted public health interventions in disadvantaged areas, such as skill development and social marketing, to reduce inequities. Additionally, while knowledge, self-efficacy, habit strength (vegetable) and injunctive norms were consistently associated with dietary intake across socio-economic strata, habit strength (fruit) and descriptive norms were weak predictors of dietary intake (data not shown). In summary, the current investigation of supermarket access found that BMI was sensitive to both geographic scale and socio-economic disadvantage, and identified walkable distances of 800 and $1000 \mathrm{~m}$ as protective of BMI for participants in disadvantaged areas. Notably, the $1000 \mathrm{~m}$ buffer corresponds to the distance to supermarkets recommended in Victorian urban planning policy ${ }^{(29)}$. This threshold informed subsequent analysis of supermarket access.

Spatial analysis of the distribution of supermarket access across Melbourne using a $1 \mathrm{~km}$ distance threshold was investigated and small geographic areas of double disadvantage were identified where high area-level disadvantage was compounded by poor supermarket access. While most households were not vulnerable, a sizeable proportion of dwellings - $12 \%$ or 187000 households across urban Melbourne, and more than 60000 households in growth area LGA - were in high disadvantaged areas and experienced poor supermarket access. When public transport opportunities were considered, most striking was the multiple disadvantages observed in older outer-ring municipalities. In addition, if frequency of public transport (particularly bus services) had been included, actual access to supermarkets would likely be 


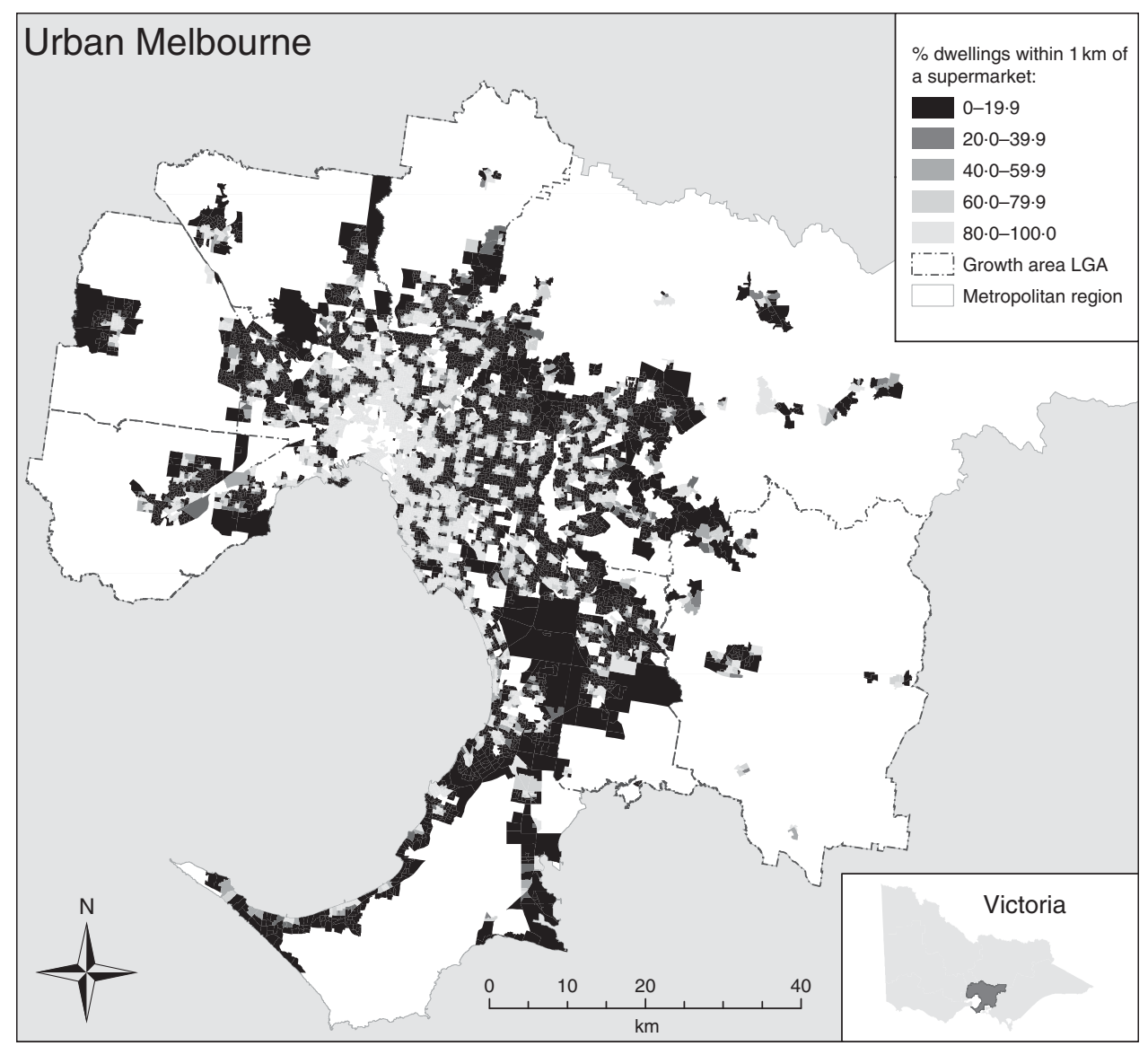

Fig. 1 Spatial distribution of supermarket access at SA1 level across urban Melbourne, Australia: percentage of dwellings within $1 \mathrm{~km}$ road network distance to the nearest supermarket (SA1, statistical areas level 1; LGA, local government area)

worse $^{(66)}$ given low public transport service levels in outer areas of Melbourne ${ }^{(67)}$.

The WHO calls on policy makers to understand and assess determinants of health inequities within cities, of global importance as cities experience rapid urbanisation and growth on urban fringes ${ }^{(68)}$. Our study evaluated the implementation of an urban planning policy providing guidance on supermarket access. In growth area LGA, only $26 \%$ of dwellings were within $1 \mathrm{~km}$ of a supermarket, far less than the 80-90\% of dwellings suggested in the policy guideline ${ }^{(29)}$. We showed that access to healthpromoting resources such as supermarkets and public transport was not evenly distributed across Melbourne, and identified geographic areas of disadvantage where inequities in access overlap. The socio-spatial patterning suggests that policy implementation should be prioritised in high disadvantaged areas across urban Melbourne, not just those within growth areas. The study highlights the utility of spatial research for identifying areas of multiple disadvantages where communities are most vulnerable and provides fine-grained data to inform urban planning policy and practice for healthier food environments.

We offer two key recommendations based on our study. First, we highlighted the importance of geographic scale in food environment research. The heterogeneity of food access measures in the literature is vast and few are theoretically or conceptually informed. It is important to identify the most appropriate geographic scale for optimal health outcomes that also considers transport mode and socio-economic areas. Second, our study showed that supermarket access had a differential impact on BMI across socio-economic strata, suggesting the importance of equity analyses in future food environment studies. The present study makes an important and timely contribution to the evidence on urban health, equity and food environments and demonstrates the potential of urban planning policy to improve health and reduce health inequities.

Our analysis had several strengths. First, we considered access to both supermarkets and fast-food chains simultaneously to account for spatial co-occurrence or clustering of fast-food outlets around supermarkets ${ }^{(41,42)}$. Second, a rigorous approach to food outlet verification was adopted; validating commercial business lists with comprehensive cross-checking against company websites, White/Yellow Pages listings and Google Street View. Sourcing accurate and comprehensive spatial data across large geographic areas is noted as a limitation in 


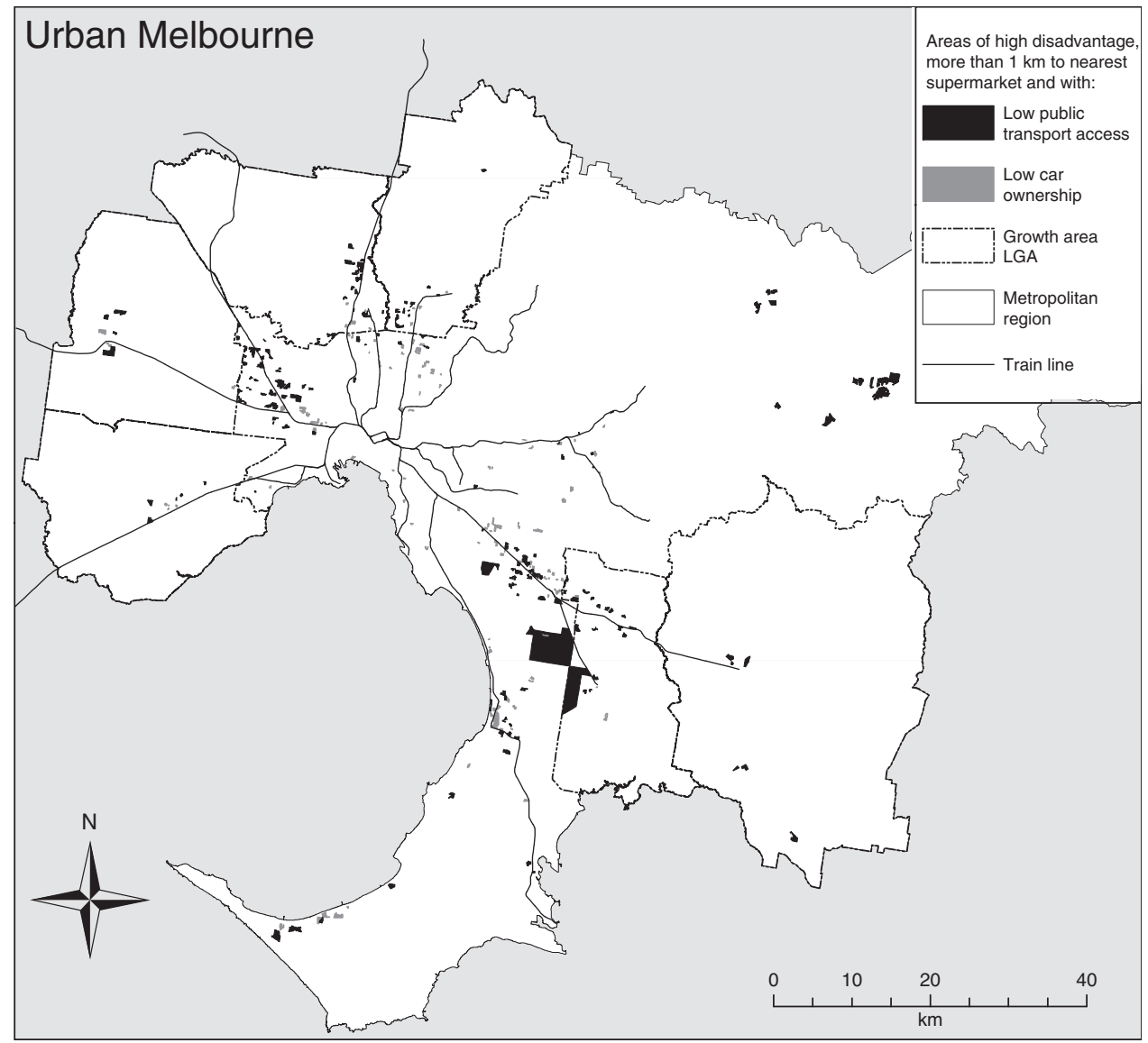

Fig. 2 Geographic areas of high disadvantage across urban Melbourne, Australia where distance to the nearest supermarket by road network is more than $1 \mathrm{~km}$ and with low public transport access or low car ownership

many studies ${ }^{(69)}$. Third, food outlet exposure data and health outcome data were contemporaneous ${ }^{(70)}$. However, there were limitations to the data used in our study. The food environment exposure variables did not include greengrocers, therefore underestimating the true food environment where people purchase vegetables and fruit; and supermarkets were used as a proxy of healthy food access even though unhealthy foods can be purchased there. Neither did we analyse in-store food environments (e.g. price, promotion of food) and other food environment exposures (e.g. near workplaces or outdoor advertising) that are also likely to influence dietary intake and BMI. Third, MB population-weighted centroids were used to calculate the spatial distribution of supermarkets and public transport access. While variation in MB size is reduced by considering only urban areas in the metropolitan region, spatial uncertainly exists. Using the interquartile range as the measure of spread, the variation in the spatial extent of the MB area was 2.09 ha. The mean and median MB area were 4.72 and 2.94 ha, respectively (data not shown). Other data limitations include selfreported rather than objectively measured health data, and cross-sectional survey data that are unable to determine causality.

\section{Conclusion}

The current study found that the local food environment differentially impacted BMI of people living in disadvantaged areas. Replication of these findings in other rapidly growing cities could assist in developing urban planning guidelines aimed at improving access to healthy food outlets across socio-economic areas. The importance of the study is that it provides evidence to guide policy interventions and suggests a way forward to reduce inequities by prioritising implementation of healthy food and public transport access policies in disadvantaged areas. The study offers useful insights into methodology and findings for other food environments studies in urban settings.

\section{Acknowledgements}

Acknowledgements: The authors acknowledge the contribution of Rebecca Roberts for creating spatial measures in this analysis and Lillian Murphy for assistance with food outlet data verification. The authors acknowledge the Department of Health and Human Services Preventive Health Survey (PHS 2012-13), Melbourne, Victoria. 
Disclaimer: The opinions and analysis in this document are those of the authors and are not those of the Department of Health and Human Services Victoria, the Victorian Government, the Secretary to the Department of Health and Human Services, or the Minister for Health. Financial support: This work was supported by the National Health and Medical Research Council (NHMRC) Centre for Excellence in Healthy Liveable Communities (grant number 1061404). M.M. is supported by the Australian Government Research Training Program Scholarship; B.G.-C. is supported by an NHMRC Senior Principal Research Fellowship (grant number 1107672); H.B. is supported by an RMIT University Vice Chancellor's Senior Research Fellowship. M.J.K. is supported by a JSPS Postdoctoral Fellowship for Research in Japan (grant number 17716). The NHMRC had no role in the design, analysis or writing of this article. Conflict of interest: None. Authorship: M.M. formulated the research question and study design, conducted the statistical and spatial analysis, and drafted the manuscript. M.J.K., H.B. and B.G.-C. contributed to the formulation of the research question and study design, data analysis and manuscript preparation. Ethics of human subject participation: This research received ethics approval from the Victorian Department of Health (No. 02/12) and The University of Melbourne Human Research Ethics Committee (Ethics ID 1441599.1).

\section{Supplementary material}

To view supplementary material for this article, please visit https://doi.org/10.1017/S1368980017002336

\section{References}

1. GBD 2013 Risk Factors Collaborators, Forouzanfar MH, Alexander L et al. (2015) Global, regional, and national comparative risk assessment of 79 behavioural, environmental and occupational, and metabolic risks or clusters of risks in 188 countries, 1990-2013: a systematic analysis for the Global Burden of Disease Study 2013. Lancet 386, 2287-2323.

2. Popkin BM (2002) The shift in stages of the nutrition transition in the developing world differs from past experiences! Public Health Nutr 5, 205-214.

3. Giles-Corti B, Vernez-Moudon A, Reis R et al. (2016) City planning and population health: a global challenge. Lancet 388, 2912-2924.

4. World Health Organization (2013) WHO Global Action Plan for the Prevention and Control of Noncommunicable Diseases 2013-2020. Geneva: WHO.

5. Caspi CE, Sorensen G, Subramanian SV et al. (2012) The local food environment and diet: a systematic review. Health Place 18, 1172-1187.

6. Zenk SN, Thatcher E, Reina M et al. (2015) Local food environments and diet-related health outcomes: a systematic review of local food environments, body weight, and other diet-related health outcomes. In Local Food Environments: Food Access in America, pp. 167-204 [K Morland, editor]. Boca Raton, FL: CRC Press.

7. Black C, Moon G \& Baird J (2014) Dietary inequalities: what is the evidence for the effect of the neighbourhood food environment? Health Place 27, 229-242.
8. Lyseen AK, Hansen HS, Harder H et al. (2015) Defining neighbourhoods as a measure of exposure to the food environment. Int J Environ Res Public Health 12, 8504-8525.

9. Ball K, Timperio A \& Crawford D (2009) Neighbourhood socioeconomic inequalities in food access and affordability. Health Place 15, 578-585.

10. Barnes TL, Colabianchi N, Hibbert JD et al. (2016) Scale effects in food environment research: Implications from assessing socioeconomic dimensions of supermarket accessibility in an eight-county region of South Carolina. Appl Geogr 68, 20-27.

11. Fan JX, Hanson HA, Zick CD et al. (2014) Geographic scale matters in detecting the relationship between neighbourhood food environments and obesity risk: an analysis of driver license records in Salt Lake County, Utah. BMJ Open 4, e005458.

12. Charreire H, Casey R, Salze P et al. (2010) Measuring the food environment using geographical information systems: a methodological review. Public Health Nutr 13, 1773-1785.

13. Morland KB (2015) Geography of local food environments: people and places. In Local Food Environments: Food Access in America, pp. 87-120 [KB Morland, editor]. Boca Raton, FL: CRC Press.

14. Feng J, Glass TA, Curriero FC et al. (2010) The built environment and obesity: a systematic review of the epidemiologic evidence. Health Place 16, 175-190.

15. Powell LM \& Han E (2011) Adult obesity and the price and availability of food in the United States. Am J Agric Econ $\mathbf{9 3}$, 378-384.

16. Beaulac J, Kristjansson E \& Cummins S (2009) A systematic review of food deserts, 1966-2007. Prev Chronic Dis 6, A105.

17. Walker RE, Keane CR \& Burke JG (2010) Disparities and access to healthy food in the United States: a review of food deserts literature. Health Place 16, 876-884.

18. Ver Ploeg M (2010) Access to Affordable and Nutritious Food: Measuring and Understanding Food Deserts and Their Consequences: Report to Congress. Washington, DC: US Department of Agriculture, Economic Research Service.

19. Cummins S \& Macintyre S (2006) Food environments and obesity - neighbourhood or nation? Int J Epidemiol 35, 100-104.

20. Macintyre S (2007) Deprivation amplification revisited; or, is it always true that poorer places have poorer access to resources for healthy diets and physical activity? Int J Behav Nutr Phys Act 4, 32 .

21. Winkler E, Turrell G \& Patterson C (2006) Does living in a disadvantaged area mean fewer opportunities to purchase fresh fruit and vegetables in the area? Findings from the Brisbane food study. Health Place 12, 306-319.

22. Burns CM \& Inglis AD (2007) Measuring food access in Melbourne: access to healthy and fast foods by car, bus and foot in an urban municipality in Melbourne. Health Place 13, 877-885.

23. Friel S, Akerman M, Hancock T et al. (2011) Addressing the social and environmental determinants of urban health equity: evidence for action and a research agenda. J Urban Health 88, 860-874.

24. Cobb LK, Appel LJ, Franco M et al. (2015) The relationship of the local food environment with obesity: a systematic review of methods, study quality, and results. Obesity (Silver Spring) 23, 1331-1344.

25. Giskes K, van Lenthe F, Avendano-Pabon M et al. (2011) A systematic review of environmental factors and obesogenic dietary intakes among adults: are we getting closer to understanding obesogenic environments? Obes Rev 12, e95-e106.

26. Drewnowski A, Aggarwal A, Tang W et al. (2016) Obesity, diet quality, physical activity, and the built environment: the need for behavioral pathways. BMC Public Health 16, 1153.

27. Murphy M, Badland $H$, Koohsari MJ et al. (2016) Indicators of a health-promoting local food environment: a conceptual framework to inform urban planning policy and practice. Health Promot J Aust 28, 82-84. 
28. Department of Environment, Land, Water and Planning (2016) Victoria in Future 2016: Population and Household Projections to 2051. Melbourne: State Government of Victoria.

29. Growth Areas Authority (2013) Precinct Structure Planning Guidelines Part Two: Preparing the Precinct Structure Plan. Melbourne: State Government of Victoria.

30. Department of Health and Human Services (2014) Preventive Health Survey (PHS) - 2012/13 and 2015/16 Overview. Melbourne: State Government of Victoria.

31. Australian Bureau of Statistics (2011) 2901.0 - Census Dictionary, 2011. Canberra: Commonwealth of Australia.

32. Kim SA, Stein AD \& Martorell R (2006) Country development and the association between parity and overweight. Int J Obes (Lond) 31, 805-812.

33. Larson MG (2006) Descriptive statistics and graphical displays. Circulation 114, 76-81.

34. Department of Health and Human Services (2016) Victorian Population Health Survey 2012, Selected Survey Findings. Melbourne: State Government of Victoria.

35. Shaikh AR, Yaroch AL, Nebeling L et al. (2008) Psychosocial predictors of fruit and vegetable consumption in adults a review of the literature. Am J Prev Med 34, 535-543.

36. Guillaumie L, Godin G \& Vezina-Im LA (2010) Psychosocial determinants of fruit and vegetable intake in adult population: a systematic review. Int J Behav Nutr Phys Act 7, 12.

37. Tak NI, te Velde SJ, Kamphuis CB et al. (2013) Associations between neighbourhood and household environmental variables and fruit consumption: exploration of mediation by individual cognitions and habit strength in the GLOBE study. Public Health Nutr 16, 505-514.

38. Ball K, Jeffery RW, Abbott G et al. (2010) Is healthy behavior contagious: associations of social norms with physical activity and healthy eating. Int J Behav Nutr Phys Act 7, 86.

39. IPAQ Group (2005) Guidelines for Data Processing and Analysis of the International Physical Activity Questionnaire (IPAQ) - Short and Long Forms. https://sites.google.com/ site/theipaq/home (accessed February 2016).

40. Department of Health (2014) Australia's Physical Activity and Sedentary Behaviour Guidelines. Canberra: Commonwealth of Australia.

41. Lamichhane AP, Warren J, Puett R et al. (2013) Spatial patterning of supermarkets and fast food outlets with respect to neighborhood characteristics. Health Place 23, 157-164.

42. Barnes TL, Freedman DA, Bell BA et al. (2016) Geographic measures of retail food outlets and perceived availability of healthy foods in neighbourhoods. Public Health Nutr 19 , 1368-1374

43. Laraia BA, Hendrickson B \& Zhang YT (2015) Local food environments and dietary intake. In Local Food Environments: Food Access in America, pp. 121-165 [K Morland, editor]. Boca Raton, FL: CRC Press.

44. Block D \& Kouba J (2006) A comparison of the availability and affordability of a market basket in two communities in the Chicago area. Public Health Nutr 9, 837-845.

45. Thornton LE, Bentley RJ \& Kavanagh AM (2009) Fast food purchasing and access to fast food restaurants: a multilevel analysis of VicLANES. Int J Behav Nutr Phys Act 6, 28.

46. Pitney Bowes Ltd (2014) Axiom Business Points. Sydney: Pitney Bowes Ltd.

47. Thornton LE, Crawford DA \& Ball K (2010) Neighbourhoodsocioeconomic variation in women's diet: the role of nutrition environments. Eur J Clin Nutr 64, 1423-1432.

48. Thornton LE, Pearce JR, Macdonald L et al. (2012) Does the choice of neighbourhood supermarket access measure influence associations with individual-level fruit and vegetable consumption? A case study from Glasgow. Int J Health Geogr 11, 29.

49. Bodor JN, Rice JC, Farley TA et al. (2010) The association between obesity and urban food environments. J Urban Health 87, 771-781.
50. Department of Economic Development, Jobs, Transport and Resources (2016) Victorian Integrated Survey of Travel and Activity (VISTA). Melbourne: State Government of Victoria.

51. Department of Sustainability and Environment (2012) Vicmap Transport. Melbourne: State Government of Victoria.

52. Australian Bureau of Statistics (2013) 2033.0.55.001 Census of Population and Housing: Socio-Economic Indexes for Areas (SEIFA), Australia, 2011. Canberra: Commonwealth of Australia.

53. Subramanian SV, Glymour MM \& Kawachi I (2007) Identifying causal ecologic effects on health: a methodological assessment. In Macrosocial Determinants of Population Health, pp. 301-331 [S Galea, editor]. New York: Springer.

54. Badland H, Turrell G \& Giles-Corti B (2013) Who does well where? Exploring how self-rated health differs across diverse people and neighborhoods. Health Place 22, 82-89.

55. Australian Bureau of Statistics (2011) Statistical Geography Fact Sheet: Statistical Areas Level 1 (SA1s). Canberra: Commonwealth of Australia.

56. Australian Bureau of Statistics (2011) Statistical Geography Fact Sheet: Mesh Blocks. Canberra: Commonwealth of Australia.

57. Thornton LE, Pearce JR \& Kavanagh AM (2011) Using Geographic Information Systems (GIS) to assess the role of the built environment in influencing obesity: a glossary. Int $J$ Behav Nutr Phys Act 8, 71.

58. Department of Environment, Land, Water and Planning (2016) Victoria Planning Provisions. Melbourne: State Government of Victoria.

59. Australian Bureau of Statistics (2016) 2011 Census of Population and Housing. Canberra: Commonwealth of Australia.

60. Gunn LD, King TL, Mavoa S et al. (2017) Identifying destination distances that support walking trips in local neighborhoods. J Transp Health 5, 133-141.

61. Commission on Social Determinants of Health (2008) Closing the Gap in a Generation: Health Equity Through Action on the Social Determinants of Health. Final Report of the Commission on Social Determinants of Health. Geneva: WHO.

62. Christian H, Bull F, Middleton N et al. (2011) How important is the land use mix measure in understanding walking behaviour? Results from the RESIDE study. Int J Behav Nutr Phys Act 8, 55.

63. Jabs J \& Devine CM (2006) Time scarcity and food choices: an overview. Appetite 47, 196-204.

64. Mackenbach JD, Burgoine T, Lakerveld J et al. (2017) Accessibility and affordability of supermarkets: associations with the DASH diet. Am J Prev Med 53, 55-62.

65. National Health and Medical Research Council (2013) Australian Dietary Guidelines: Providing the Scientific Evidence for Healthier Australian Diets. Canberra: NHMRC.

66. Bader MDM, Purciel M, Yousefzadeh P et al. (2010) Disparities in neighborhood food environments: implications of measurement strategies. Econ Geogr 86, 409-430.

67. Currie G, Richardson T, Smyth P et al. (2010) Investigating links between transport disadvantage, social exclusion and well-being in Melbourne - updated results. Res Transport Econ 29, 287-295.

68. World Health Organization (2010) Hidden Cities: Unmasking and Overcoming Health Inequities in Urban Settings. Geneva: WHO.

69. Moore LV \& Diez-Roux AV (2014) Measurement and analytical issues involved in the estimation of the effects of local food environments on health behaviors and health outcomes. In Local Food Environments: Food Access in America, pp. 205-230 [K Morland, editor]. Boca Raton, FL: CRC Press.

70. Thornton LE \& Kavanagh AM (2010) Understanding the local food environment and obesity. In Geographies of Obesity: Environmental Understandings of the Obesity Epidemic, pp. 79-110 [J Pearce and K Witten, editors]. Farnham: Ashgate Publishing Limited. 